\title{
ASYMPTOTICS OF THE ENTIRE FUNCTIONS WITH $v$-DENSITY OF ZEROS ALONG THE LOGARITHMIC SPIRALS
}

\begin{abstract}
Let $v$ be the growth function such that $r v^{\prime}(r) / v(r) \rightarrow 0$ as $r \rightarrow+\infty, l_{\varphi}^{c}=\left\{z=t e^{i(\varphi+c \ln t)}, 1 \leqslant\right.$ $t<+\infty\}$ be the logarithmic spiral, $f$ be the entire function of zero order. The asymptotics of $\ln f\left(r e^{i(\theta+c \ln r)}\right)$ along ordinary logarithmic spirals $l_{\theta}^{c}$ of the function $f$ with $v$-density of zeros along $l_{\varphi}^{c}$ outside of the $C_{0}$-set is found. The inverse statement is true just in case zeros of $f$ are placed on the finite logarithmic spirals system $\Gamma_{m}=\bigcup_{j=0}^{m} l_{\theta_{j}}^{c}$.

Key words and phrases: entire function, density of zeros, logarithmic spiral.
\end{abstract}

Ivan Franko National University, 1 Universytetska str., 79000, Lviv, Ukraine

E-mail: mykola.zabolotskyy@lnu.edu.ua (Zabolotskyi M.V.), yuliya.basyuk.92@gmail.com (Basiuk Yu.V.)

\section{INTRODUCTION}

The issues related to the study of behavior of entire functions along the logarithmic spirals were considered in [1-4,6]. In particular, Macintyre [6] introduced the notion of an indicator along the logarithmic spiral and generalized the concept of associated function. Kennedy [3] generalized the concept of Mittag - Leffler function on the curvilinear area. Valiron-type and Valiron-Titchmarsh-type theorems for entire functions of positive order with zeros on the logarithmic spiral were proved by Balašov [2] and Kheifits [4] correspondingly. The relation between regular behavior of logarithm of modulus of entire function $f$ of positive order along the curves of regular rotation (in particular, the logarithmic spirals) and existance of density of zeros of $f$ along these curves was investigated in [1]. The results of [1] generalize the wellknown Levin and Pfluger research of entire functions of completely regular growth (see, for example, [5, p. 118-122; p. 199]).

In this paper we study issues that similar to ones considered in [1] for entire functions of zero order.

\section{SECTION WITH RESULTS}

For $c \in \mathbb{R}, \varphi \in[-\pi ; \pi)$ we denote by $l_{\varphi}^{c}(a, r)=\left\{z: z=t e^{i(\varphi+c \ln t)}, a \leqslant t<r\right\}, l_{\varphi}^{c}(1,+\infty)=$ $l_{\varphi}^{c}$ the logarithmic spiral, $D^{c}(r ; \alpha, \beta)=\bigcup_{\alpha \leqslant \varphi<\beta} l_{\varphi}^{c}(1, r)$ the curvilinear sector, $-\pi \leqslant \alpha<\beta<\pi$.

Let $L$ be the set of all growth functions $v$ such that $r v^{\prime}(r) / v(r) \rightarrow 0$ as $r \rightarrow+\infty$ where growth function $v:[0 ;+\infty) \rightarrow \mathbb{R}_{+}$is a continuously differentiable increasing to $+\infty$ function. It is clear that a set $L$ coincides with accuracy to equivalent functions with a set of slow growing 
functions in the sense of Karamata ([7, p. 15]). For $v \in L$ we denote by $H_{0}(v)$ the class of entire functions $f$ of zero order that satisfy the condition $n(r)=O(v(r)), r \rightarrow+\infty$, where $n(r)=n(r, 0, f)$ is counting function of zeros $\left(a_{n}\right)_{n=1}^{+\infty}$ of function $f$.

We say that zeros of the function $f \in H_{0}(v)$ have $v$-density $\Delta^{c}(\alpha, \beta)$ along logarithmic spirals $l_{\varphi}^{c}$ if the limit

$$
\lim _{r \rightarrow \infty} \frac{n^{c}(r ; \alpha, \beta)}{v(r)}=\Delta^{c}(\alpha, \beta)
$$

exists for all $\alpha, \beta \in \mathbb{R}, 0<\beta-\alpha \leqslant 2 \pi$ with the exception, perhaps, of $\alpha$ or $\beta$ belongs to some countable set $\mathcal{N}$, where $n^{c}(r ; \alpha, \beta)$ is a number of zeros of the function $f$ in $D^{c}(r ; \alpha, \beta)$.

The equality $\Delta^{c}(\varphi)=\Delta^{c}\left(\varphi_{1}, \varphi\right)$ for a fixed $\varphi_{1} \notin \mathcal{N}$ defines on the segment $\left[\varphi_{1}, \varphi_{1}+2 \pi\right]$ a non-decreasing function $\Delta^{c}(\varphi)$ which we extend on $\mathbb{R}$ by the rule $\Delta^{c}(\varphi+2 \pi)-\Delta^{c}(\varphi)=$ $\Delta^{c}\left(\varphi_{1}+2 \pi\right)-\Delta^{c}\left(\varphi_{1}\right)$.

The logarithmic spiral $l_{\theta}^{c}$ satisfying the condition

$$
\lim _{h \rightarrow 0+r \rightarrow+\infty} \frac{n^{c}(r ; \theta-h, \theta+h)}{v(r)}=0
$$

is called ordinary for $f \in H_{0}(v)$. The other logarithmic spirals are called exceptional. It follows from monotonicity of the function $\Delta^{c}(\varphi)$ that the set of exceptional logarithmic spirals is no more than countable if zeros of $f \in H_{0}(v)$ have $v$-density $\Delta^{c}(\alpha, \beta)$ along $l_{\varphi}^{c}$.

Denote by $\ln \left(1-\frac{z}{a_{n}}\right), a_{n} \in l_{\theta}^{c}$ the single-valued in the domain $D\left(l_{\theta}^{c}\right)=\mathbb{C} \backslash l_{\theta}^{c}\left(\left|a_{n}\right|,+\infty\right)$ branch of multi-valued function $\operatorname{Ln}\left(1-\frac{z}{a_{n}}\right)$ such that $\left.\ln \left(1-\frac{z}{a_{n}}\right)\right|_{z=0}=0$. Let

$$
f(z)=\prod_{n=1}^{+\infty}\left(1-\frac{z}{a_{n}}\right) \in H_{0}(v) \text {. }
$$

Then

$$
\ln f(z)=\sum_{n=1}^{+\infty} \ln \left(1-\frac{z}{a_{n}}\right), z \in \mathbb{C} \backslash \bigcup_{n=1}^{+\infty} l_{\varphi_{i}}^{c}\left(r_{i},+\infty\right),
$$

where $r_{i}$ is the minimum module of zeros $a_{i}$ of $f$ that lie on the logarithmic spiral $l_{\varphi_{i}}^{c}, \varphi_{i}=$ $\arg a_{i} \in[-\pi, \pi)$.

We call a set $E \in \mathbb{C}$ the $C_{0}$-set if it can be covered by a system of circles $\left\{z:\left|z-a_{k}\right|<\right.$ $\left.r_{k}\right\}, k \in \mathbb{N}$ such that $\sum_{\left|a_{k}\right| \leqslant r} r_{k}=o(r), r \rightarrow+\infty$.

We write $\hat{h}(\theta ; \psi)$ for the $2 \pi$-periodic extension of the function $h(\theta ; \psi)=\theta-\psi-\pi$ from $(\psi ; \psi+2 \pi)$ to $\mathbb{R},-\pi \leqslant \psi<\pi$. Note $N(r)=N(r, 0, f)=\int_{0}^{r} \frac{n(t)}{t} d t$,

$$
H_{f}^{c}(\theta)=\int_{\theta-2 \pi}^{\theta}(\theta-\psi-\pi) d \Delta^{c}(\psi)=\int_{-\pi}^{\pi} \hat{h}(\theta ; \psi) d \Delta^{c}(\psi) .
$$

Theorem 1. Let $v \in L, f \in H_{0}(v)$, zeros of $f$ have $v$-density $\Delta^{c}(\alpha, \beta)$ along $l_{\varphi}^{c}$. Then there is a $C_{0}$-set $E$ such that the following asymptotic relation holds $(|z|=r)$ :

$$
\ln f(z)=(1+i c) N(r)+i H_{f}^{c}(\theta) v(r)+o(v(r)), z \in l_{\theta}^{c}, z \notin E,
$$

where $l_{\theta}^{c}$ is ordinary logarithmic spiral. 
Let $\Gamma_{m}=\bigcup_{j=1}^{m} l_{\theta^{\prime}}^{c}-\pi \leqslant \theta_{1}<\ldots<\theta_{m}<\pi$ be a finite system of logarithmic spirals, $\theta_{m+1}=\theta_{1}+2 \pi$.

Theorem 2. Let $v \in L, f \in H_{0}(v)$, zeros of $f$ lie on $\Gamma_{m}, H$ be a piecewise continuous on $[-\pi, \pi)$ function. If for any $\delta>0$ the following asymptotic relation

$$
\ln f\left(r e^{i(\theta+c \ln r)}\right)=(1+i c) N(r)+i H(\theta) v(r)+o(v(r)), r \rightarrow \infty
$$

holds uniformly with respect to $\theta \in[-\pi, \pi) \backslash \bigcup_{j=1}^{m+1}\left(\theta_{j}-\delta ; \theta_{j}+\delta\right)$, then zeros of $f$ have $v$-density $\Delta^{c}(\alpha, \beta)$ along $l_{\varphi}^{c}$.

Remark. The condition that zeros of $f \in H_{0}(v)$ lie on a finite system of logarithmic spirals $\Gamma_{m}$ is significant in Theorem 2. In the general case of zeros arrangement the statement of Theorem 2 is wrong (see [8] in case $c=0$ ).

\section{THE PROOF OF RESULTS}

At first we present the lemmas that will be used in the proof of the theorems.

Lemma 1 ([11]). Let $\Delta>0, v \in L, f \in H_{0}(v)$, zeros of $f$ lie on the logarithmic spiral $l_{\psi}^{c}, \psi \in \mathbb{R}$,

$$
n(r)=(1+o(1)) \Delta v(r), r \rightarrow+\infty .
$$

Then for $\theta \in \mathbb{R} \backslash\{\psi+2 \pi k: k \in \mathbb{Z}\}$ the following asymptotic relation holds:

$$
\ln f\left(r e^{i(\theta+c \ln r)}\right)=(1+i c) N(r)+i \Delta \hat{h}(\theta ; \psi) v(r)+o(v(r)), r \rightarrow \infty,
$$

moreover, relation (5) is uniform with respect to $\theta \in[\psi+\delta ; \psi+2 \pi-\delta], 0<\delta<1$.

Lemma 2. Let $f$ has the form defined in (1), zeros of $f$ have $v$-density $\Delta^{c}(\alpha, \beta)$ along $l_{\varphi}^{c}, \varepsilon>0$ is arbitrary number. Then there exist $\delta>0$ and a $C_{0}$-set $E$ such that for all ordinary logarithmic spirals $l_{\theta}^{c}$ of the function $f$ the following inequality holds:

$$
\left|\ln f(z)-\ln f^{\delta}(z)\right|<\varepsilon v(r), z \in l_{\theta}^{c}, z \notin E,
$$

where $f^{\delta}(z)=\prod_{n=1}^{+\infty}\left(1-\frac{z}{a_{n}^{\prime}}\right),\left|a_{n}^{\prime}\right|=\left|a_{n}\right|,\left|\arg a_{n}-\arg a_{n}^{\prime}\right|<\delta$.

The proof of the Lemma 2 follows from the considerations similar to [5, p. 132-133], [1, p. 352-353] and Theorem 1 from [10].

We say that a set $F \subset \mathbb{R}_{+}$is $E_{0}$-set if $F$ is a measurable and mes $(E \cap[0, r])=o(r), r \rightarrow+\infty$.

In view of Lemmas 4 and 5 from [9], we get

Lemma 3. Let $\theta \in[-\pi, \pi), v \in L, f \in H_{0}(v), \delta>0$. Then there exists a $E_{0}$-set $F$ such that

$$
r \int_{\theta-\delta}^{\theta+\delta}\left|\frac{f^{\prime}\left(r e^{i \varphi}\right)}{f\left(r e^{i \varphi}\right)}\right| d \varphi=O(v(r))\left(\delta+\delta \ln \left(1+\frac{1}{\delta}\right)\right), r \rightarrow+\infty, r \notin F .
$$


Proof of Theorem 1. Let $\varepsilon>0$ is given arbitrary number, function $H_{f}^{c}(\theta)$ defined by formula (2). Choose $\delta>0$ such that the integral sum

$$
S_{m}(\theta)=\sum_{j=0}^{m-1} \hat{h}\left(\theta ; \psi_{j}\right)\left(\Delta^{c}\left(\psi_{j+1}\right)-\Delta^{c}\left(\psi_{j}\right)\right),
$$

where $-\pi=\psi_{0}<\psi_{1}<\ldots<\psi_{m-1}<\psi_{m}=\pi, \max _{0 \leqslant j \leqslant m-1}\left|\psi_{j+1}-\psi_{j}\right|<\delta$, satisfies the inequality

$$
\left|H_{f}^{c}(\theta)-S_{m}(\theta)\right|<\frac{\varepsilon}{3}
$$

Then take numbers $a_{k}^{\prime}$ such that $\left|a_{k}^{\prime}\right|=\left|a_{k}\right|, a_{k}^{\prime} \in l_{\psi_{j}}^{c}$ if $a_{k} \in l_{\psi^{\prime}}^{c} \psi_{j} \leqslant \psi<\psi_{j+1}(j=$ $0,1, \ldots, m-1)$ and build the function $f^{\delta}(z)$. Applying Lemma 2 we obtain that there exist $\delta>0$ and $C_{0}$-set $E_{1}$ such that for all ordinary logarithmic spirals $l_{\theta}^{c}$ of $f$ and $f^{\delta}$ the following inequality holds:

$$
\left|\ln f(z)-\ln f^{\delta}(z)\right|<\frac{\varepsilon}{3} v(r), z \notin E_{1}, z \in l_{\theta}^{c} .
$$

Zeros of $f^{\delta}(z)$ lie on a finite system of logarithmic spirals $\Gamma_{m}$ so $f^{\delta}(z)$ can be depicted as a product of $m$ entire functions such that zeros of each function lie on a single logarithmic spiral $l_{\psi_{j}}^{c}$. From Lemma 1 (see (5)) we get that inequality

$$
\left|\frac{\ln f^{\delta}(z)-(1+i c) N(r)}{v(r)}-i S_{m}(\theta)\right|<\varepsilon, z \in l_{\theta}^{c}
$$

holds uniformly with respect to $\theta \in \mathbb{R} \backslash \bigcup_{j=1}^{m}\left(\psi_{j}-\delta ; \psi_{j}+\delta\right)$, where $\delta>0$ is an arbitrary number.

Further taking into account (6), (7) we obtain that for $z \notin E_{1}, z \in l_{\theta}^{c}, \theta \in \mathbb{R} \backslash \bigcup_{j=1}^{m}\left(\psi_{j}-\right.$ $\left.\delta ; \psi_{j}+\delta\right)$ the following inequality holds:

$$
\left|\frac{\ln f(z)-(1+i c) N(r)}{v(r)}-i H_{f}^{c}(\theta)\right|<\varepsilon
$$

Choosing another segmentation of $[-\pi ; \pi]$ by points $\left(\psi_{j}^{\prime}\right)_{j=0}^{m},\left|\psi_{j+1}^{\prime}-\psi_{j}^{\prime}\right|<\delta$ such that intervals $\left(\psi_{j}^{\prime}-\delta ; \psi_{j}^{\prime}+\delta\right)$ do not have the mutual points with intervals $\left(\psi_{j}-\delta ; \psi_{j}+\delta\right)$, we get that (8) holds for $z \notin E_{2}, z \in l_{\theta}^{c}, \theta \in \mathbb{R} \backslash \bigcup_{j=1}^{m}\left(\psi_{j}^{\prime}-\delta ; \psi_{j}^{\prime}+\delta\right)$, where $E_{2}$ is some $C_{0}$-set.

This yields that (3) holds for all ordinary logarithmic spirals $l_{\theta}^{c}$ of function $f$. So Theorem 1 is proved.

Proof of Theorem 2. Let $v \in L, \Omega=\left\{\left|a_{n}\right|: n \in \mathbb{N}\right\}, a_{n}$ be zeros of $f \in H_{0}(v)$ that lie on a finite system of logarithmic spirals $\Gamma_{m}=\bigcup_{j=1}^{m} l_{\theta_{j^{\prime}}}^{c}-\pi \leqslant \theta_{1}<\ldots<\theta_{m}<\pi$. Set

$$
\partial D^{c}(r ; \alpha, \beta)=l_{\alpha}^{c}(1, r) \cup \Gamma(r ; \alpha, \beta) \cup\left(l_{\beta}^{c}(1, r)\right)^{-1} \cup(\Gamma(1 ; \alpha, \beta))^{-1},
$$

where $r \notin \Omega,-\pi \leqslant \theta_{k_{0}-1}<\alpha<\theta_{k_{0}}<\ldots<\theta_{s_{0}}<\beta<\theta_{s_{0}+1}<\pi$,

$$
\Gamma(\tau ; \alpha, \beta)=\left\{z=\tau e^{i(\varphi+c \ln \tau)}: \alpha \leqslant \varphi \leqslant \beta\right\} .
$$


Since $d z=(1+i c) e^{i(\varphi+c \ln t)} d t$ for $l_{\theta}^{c}(1, r)$ then with the notation

$$
F(\tau, \varphi)=\tau e^{i(\varphi+c \ln \tau)} \frac{f^{\prime}\left(\tau e^{i(\varphi+c \ln \tau)}\right)}{f\left(\tau e^{i(\varphi+c \ln \tau)}\right)}
$$

using Residue theorem we have

$$
\begin{aligned}
2 \pi i n^{c}(r ; \alpha, \beta) & =\int_{\partial D^{c}(r ; \alpha, \beta)} \frac{f^{\prime}(z)}{f(z)} d z=\left(\int_{l_{\alpha}^{c}(1, r)}+\int_{\Gamma(r ; \alpha, \beta)}-\int_{l_{\beta}^{c}(1, r)}-\int_{\Gamma(1 ; \alpha, \beta)}\right) \frac{f^{\prime}(z)}{f(z)} d z \\
= & (1+i c) \int_{1}^{r}\left(\frac{F(t, \alpha)}{t}-\frac{F(t, \beta)}{t}\right) d t+\int_{\alpha}^{\beta}(F(r, \theta)-F(1, \theta)) i d \theta \\
= & \ln f\left(r e^{i(\alpha+c \ln r)}\right)-\ln f\left(r e^{i(\beta+c \ln r)}\right) \\
& +\left(\int_{\alpha}^{\theta_{k_{0}}-\delta}+\sum_{j=k_{0}}^{s_{0}-1} \int_{\theta_{j}+\delta}^{\theta_{j+1}-\delta}+\int_{\theta_{s_{0}}+\delta}^{\beta}+\sum_{j=k_{\theta_{j}}-\delta}^{s_{0}} \int_{\theta_{j}+\delta}\right) F(r, \theta) i d \theta+C,
\end{aligned}
$$

where $C=-\ln f\left(e^{i \alpha}\right)+\ln f\left(e^{i \beta}\right)-\int_{\alpha}^{\beta} F(1, \theta) i d \theta, 0<\delta<\min \left\{\frac{\theta_{k_{0}}-\alpha}{2}, \frac{\beta-\theta_{s_{0}}}{2}, \frac{\theta_{j+1}-\theta_{j}}{2}\right\}$, $j=\overline{k_{0}, s_{0}-1}$.

$$
\theta_{j+1}-\delta
$$

Taking account of $\int_{\theta_{j}+\delta} F(r, \theta) i d \theta=\ln f\left(r e^{i\left(\theta_{j+1}-\delta+c \ln r\right)}\right)-\ln f\left(r e^{i\left(\theta_{j}+\delta+c \ln r\right)}\right)$, from (9) we obtain

$$
\begin{aligned}
2 \pi i n^{c}(r ; \alpha, \beta)= & \sum_{j=k_{0}}^{s_{0}}\left(\ln f\left(r e^{i\left(\theta_{j}-\delta+c \ln r\right)}\right)-\ln f\left(r e^{i\left(\theta_{j}+\delta+c \ln r\right)}\right)\right) \\
& +\sum_{j=k_{0}}^{s_{0}} \int_{\theta_{j}-\delta}^{\theta_{j}+\delta} F\left(r e^{i(\theta+c \ln r)}\right) i d \theta=\sum_{1}+\sum_{2} .
\end{aligned}
$$

Applying (4) we get

$$
\sum_{1}=i \sum_{j=k_{0}}^{s_{0}}\left(H\left(\theta_{j}-\delta\right)-H\left(\theta_{j}+\delta\right)\right) v(r)+o(v(r)), r \rightarrow \infty
$$

In view of Lemma 3 , there exist $E_{0}$-sets $F_{j}$ such that $\left(j=\overline{k_{0}, s_{0}}\right)$

$$
\begin{aligned}
\left|\int_{\theta_{j}-\delta}^{\theta_{j}+\delta} F\left(r e^{i(\theta+c \ln r)}\right) i d \theta\right| & \leqslant r \int_{\theta_{j}-\delta}^{\theta_{j}+\delta}\left|\frac{f^{\prime}\left(r e^{i(\theta+c \ln r)}\right)}{f\left(r e^{i(\theta+c \ln r)}\right)}\right| d \theta=r \int_{\theta_{j}-\delta}^{\theta_{j}+\delta}\left|\frac{f^{\prime}\left(r e^{i \varphi}\right)}{f\left(r e^{i \varphi}\right)}\right| d \varphi \\
& =O(v(r))\left(\delta+\delta \ln \left(1+\frac{1}{\delta}\right)\right), r \rightarrow+\infty, r \notin F_{j} .
\end{aligned}
$$


So,

$$
\left|\sum_{2}\right| \leqslant K_{1}(v(r))\left(\delta+\delta \ln \left(1+\frac{1}{\delta}\right)\right), r \rightarrow+\infty, r \notin F
$$

where $F=\bigcup_{j=k_{0}}^{s_{0}} F_{j}$ is a $E_{0}$-set, $K_{1}$ is some constant.

Combining the last inequalities and (10) yields

$$
\lim _{\substack{r \rightarrow+\infty \\ r \notin E}} \frac{n^{c}(r ; \alpha, \beta)}{v(r)}=\frac{1}{2 \pi} \sum_{j=k_{0}}^{s_{0}}\left(H\left(\theta_{j}-\delta\right)-H\left(\theta_{j}+\delta\right)\right)+K_{2}\left(\delta+\delta \ln \left(1+\frac{1}{\delta}\right)\right) .
$$

Directing $\delta$ to $0+$ gives

$$
\lim _{\substack{r \rightarrow+\infty \\ r \notin E}} \frac{n^{c}(r ; \alpha, \beta)}{v(r)}=\frac{1}{2 \pi} \sum_{j=k_{0}}^{s_{0}}\left(H\left(\theta_{j}-0\right)-H\left(\theta_{j}+0\right)\right):=\Delta(\alpha, \beta) .
$$

Whereas $F$ is $E_{0}$-set, then any interval $(R,(1+\eta) R), \eta>0$, includes points that are not in $F$. Due to the monotonicity of the function $n^{c}(r ; \alpha, \beta)$ with respect to $r$ for $r>R_{0}$ we can assert that

$$
\frac{n^{c}\left(r_{1} ; \alpha, \beta\right)}{v\left(r_{1}\right)} \frac{v\left(r_{1}\right)}{v(r)} \leqslant \frac{n^{c}(r ; \alpha, \beta)}{v(r)} \leqslant \frac{n^{c}\left(r_{2} ; \alpha, \beta\right)}{v\left(r_{2}\right)} \frac{v\left(r_{2}\right)}{v(r)}
$$

where $r(1-\eta)<r_{1}<r<r_{2}<(1+\eta) r, r_{1}, r_{2} \notin F$.

Since $v\left(r_{2}\right) \sim v(r) \sim v\left(r_{1}\right), r \rightarrow \infty$, the last relation yields

$$
\lim _{r \rightarrow \infty} \frac{n^{c}(r ; \alpha, \beta)}{v(r)}=\Delta(\alpha, \beta) .
$$

Theorem 2 is proved.

\section{REFERENCES}

[1] Balašov S.K. On entire functions of completely regular growth along curves of regular rotation. Math. USSR-Izv. 1976, 10 (2), 321-338. doi:10.1070/IM1976v010n02ABEH001691 (translation of Math. USSR-Izv. 1976, 30 (2), 338-354 (in Russian)).

[2] Balašov S.K. On entire functions of finite order with zeros on curves of regular rotation. Math. USSR-Izv. 1973, 7 (3), 601-627. doi:10.1070/IM1973v007n03ABEH001963 (translation of Math. USSR-Izv. 197337 (3), $603-629$ (in Russian)).

[3] Kennedy P. A class of integral functions bounded on certain curves. Proc. Lond. Math. Soc. 1956, 3 (6), $518-547$. doi:10.1112/plms/s3-6.4.518

[4] Kheifits A.I. Analogue of the Valiron-Titchmarsh theorem for entire functions with roots on a logarithmic spiral. Izv. Vyssh. Uchebn. Zaved. Mat. 1980, 12, 74-75. (in Russian)

[5] Levin B.Ja. Distribution of Zeros of Entire Functions. State publishing house of technical and theoretical literature, Moscow, 1956. (in Russian)

[6] Macintyre A. Laplace's transformation and integral functions. Proc. Lond. Math. Soc. 1939, 45 (2), 1-20. doi:10.1112/plms/s2-45.1.1

[7] Seneta E. Regularly varying functions. Springer-Verlag, Berlin- Heidelberg - New York, 1976. 
[8] Zabolotskii M. An example of entire function of strongly regular growth. Mat. Stud. 2000, 13 (2), 145-148.

[9] Zabolotskii N.V. Strongly regular growth of entire functions of order zero. Math. Notes. 1998, 63 (2), $172-182$. (translation of Math. Notes. 1998, 63 (2), 196-208. doi:10.4213/mzm1266)

[10] Zabolotskyi M.V, Basiuk Y.V. Proximity of the entire functions of zero order with v-density of zeros. Visnyk of the Lviv Univ. Series Mech. Math. 2017, 84, 80-86.

[11] Zabolotskyi M.V, Basiuk Y.V., Tarasyuk S.I. Entire functions of zero order with zeros on the logarithmic spiral. Ukrain. Mat. Zh. 2018, 70 (7), 923-932. (in Ukrainian)

Received 20.09.2018

Заболоцький М.В., Басюк Ю.В. Асимптотика чулих функцій з v-цільністю нулів вздовж логарифміиних спіралей // Карпатські матем. публ. - 2019. - Т.11, №1. - С. 26-32.

Нехай функція зростання $v$ така, що $r v^{\prime}(r) / v(r) \rightarrow 0$ при $r \rightarrow+\infty, l_{\varphi}^{c}=\left\{z=t e^{i(\varphi+c \ln t)}, 1 \leqslant\right.$ $t<+\infty\}$ - логарифмічна спіраль, $f$ - ціла функція нульового порядку. За умови існування $v$-щільності нулів $f$ вздовж $l_{\varphi}^{c}$ знайдено асимптотику $\ln f\left(r e^{i(\theta+c \ln r)}\right)$ вздовж звичайних логарифмічних спіралей $l_{\theta}^{c}$ функції $f$ зовні $C_{0}$-множини. Показано, шо обернене до цього твердження правильне лише у випадку розташування нулів $f$ на скінченній системі логарифмічних спіралей $\Gamma_{m}=\bigcup_{j=0}^{m} l_{\theta_{j}}^{c}$.

Ключові слова і фрази: ціла функція, щільність нулів, логарифрмічна спіраль. 